\title{
Olfactory learning of plant genotypes by a polyphagous insect predator
}

\author{
Robert Glinwood · Elham Ahmed · Erika Qvarfordt • \\ Velemir Ninkovic
}

Received: 2 November 2010/Accepted: 15 December 2010/Published online: 4 January 2011

(C) The Author(s) 2010. This article is published with open access at Springerlink.com

\begin{abstract}
Olfactory learning may allow insects to forage optimally by more efficiently finding and using favourable food sources. Although olfactory learning has been shown in bees, insect herbivores and parasitoids, there are fewer examples from polyphagous predators. In this study, olfactory learning by a predatory coccinellid beetle is reported for the first time. In laboratory trials, adults of the aphidophagous ladybird Coccinella septempunctata did not prefer the odour of one aphid-infested barley cultivar over another. However, after feeding on aphids for $24 \mathrm{~h}$ on a cultivar, they preferred the odour of that particular cultivar. The mechanism appeared to be associative learning rather than sensitisation. Although inexperienced ladybirds preferred the odour of an aphid-infested barley cultivar over uninfested plants of the same cultivar, after feeding experience on a different cultivar this preference disappeared. This may indicate the acquisition and replacement of olfactory templates. The odour blends of the different aphid-infested barley cultivars varied qualitatively and quantitatively, providing a potential basis for olfactory discrimination by the ladybird. The results show that predatory coccinellids can learn to associate the odour of aphid-infested plants with the presence of prey, and that this olfactory learning ability is sensitive enough to discriminate variability between different genotypes of the same plant.
\end{abstract}

Keywords Associative learning - Olfactory learning . Ladybird · Coccinellid

Communicated by Richard Karban.

R. Glinwood $(\bowtie) \cdot$ E. Ahmed · E. Qvarfordt · V. Ninkovic Department of Ecology, Swedish University of Agricultural Sciences, Box 7044, 75007 Uppsala, Sweden

e-mail: robert.glinwood@slu.se

\section{Introduction}

The ability to learn may allow animals to optimise resource use in environments with a moderate degree of unpredictability. Learning has been demonstrated in a range of arthropods including social insects (Farina et al. 2005), herbivores (Dukas and Bernays 2000; Egas and Sabelis 2001; Shikano and Isman 2009), parasitoids (Stireman 2002; van Nouhuys and Kaartinen 2008) and predators (Guillette et al. 2009; Rahmani et al. 2009). Predatory arthropods use chemical information in host searching, particularly in the form of volatile cues released by plants in response to feeding by their herbivore prey (Dicke 2009; Heil 2008), and they can learn to associate odours with the occurrence of prey (De Boer and Dicke 2006; Dukas 2008; Vet and Dicke 1992). The volatile blends released by plants can vary widely between different combinations of plant and herbivore (Van Den Boom et al. 2004), between different herbivores on the same plant species (Leitner et al. 2005) and even between the same herbivore on different genotypes of the same plant species (Degen et al. 2004). This variability may inform foraging predators about the identity and quality of the host plant and prey, but it may limit the usefulness of innate and immutable responses to volatile blends, favouring learning instead.

In olfactory associative learning, an odour cue is temporally paired with a conditional stimulus such as feeding or contact with the prey or its products (Papaj and Prokopy 1989; Vet and Dicke 1992). For a predator that can exploit a range of resources that vary in quality and availability both in time and space, associative learning of odour cues may increase the efficiency with which the most current favourable resource is found and exploited. Most studies on olfactory learning in arthropods have focused on honey bees, fruit flies and parasitoids (Dukas 2008; Vet and Dicke 
1992) and, aside from certain predatory mites that have been extensively studied (De Boer and Dicke 2006), there are relatively few examples from predators.

The seven-spot ladybird, Coccinella septempunctata L., is a polyphagous predator that combines a broadly generalist diet with temporal focus on aphids, an essential food needed to achieve maximum fecundity (Evans et al. 1999; Hodek and Honek 1996). Ladybird foraging involves navigation through a complex landscape in which suitable, high quality food sources are found and exploited when they are available (Pettersson et al. 2005). The occurrence of aphids is patchy, varying in time and space in a way that is partly, but not completely, predictable by habitat and season. Optimal foraging models predict that predators should maximise their encounters with the most profitable prey (Stephens and Krebs 1986), so the ability to learn cues associated with high quality aphid prey when it is available should enhance the foraging efficiency of $C$. septempunctata. Indeed, there is evidence of learning by predatory coccinellids in relation to experience and prey selection (Boivin et al. 2010; Dejean et al. 2003; Ettifouri and Ferran 1993; Guershon and Gerling 2006; Houck 1986), but olfactory learning has not been reported.

Predatory coccinellids, including C. septempunctata, are attracted by odour cues associated with aphid infested plants (Bahlai et al. 2008; Hatano et al. 2008; Ninkovic et al. 2001; Zhu and Park 2005), and may also use volatile cues as indicators of habitat quality (Glinwood et al. 2009; Ninkovic et al. 2010; Ninkovic and Pettersson 2003; Pettersson et al. 2008; Sarmento et al. 2007). By learning to associate the odour of infested plants with the presence of aphids, the ladybird could increase its foraging success by more effectively finding or remaining in plant patches where aphids are present. Most studies of olfactory learning in arthropods have either compared naïve individuals with those experienced on a single plant species, or compared those experienced on plant/prey combinations that vary in quality. In the current study, we used different genotypes of the same plant species, barley (Hordeum vulgare), infested with the same aphid, bird cherry-oat aphid Rhopalosiphum padi L., to address two questions: (1) can the olfactory preference of $C$. septempunctata be modified by experience, and (2) can $C$. septempunctata learn to discriminate between the odour of aphid-infested plants of different genotypes of the same host plant species?

\section{Materials and methods}

Plants

Barley plants, $H$. vulgare L. (cvs. Barke, Lina, Frieda and Prestige) were grown in plastic pots $(9 \times 9 \times 7 \mathrm{~cm})$ in potting soil (Hasselfors Garden, Sweden) with 10 plants per pot. We chose these barley cultivars because preliminary work had indicated differences in the volatile profiles of aphid-infested plants. Plants were at the early two-leaf stage (7 days after planting) when they were infested with aphids, and at the mid-two-leaf stage (11 days after planting) at the beginning of ladybird exposure or behavioural bioassay. Thus, all plants for ladybird experience and olfactometry had been infested with aphids for 4 days. Separate pots of plants were used for ladybird exposures and subsequent bioassays. Plants were grown in a glasshouse at $20 \pm 1^{\circ} \mathrm{C}$, with a L16:D8 light cycle with supplementary lighting.

Insects

Bird cherry-oat aphid R. padi was reared on barley (cv. Golf) in multi-clonal cultures in a glasshouse under the same conditions as the plants. Aphids used to infest plants were wingless, mixed-instar individuals. To obtain infested plants for ladybird experience, olfactory tests and volatile collection, an average of 20 aphids per plant were placed carefully on the soil from where they climbed and settled on the barley plants. Plants were enclosed in perforated plastic bags $(33 \times 50 \mathrm{~cm}$, Crispac; Baumann, Waldenburg, Germany) and kept in a glasshouse at $20 \pm 1^{\circ} \mathrm{C}$, with a L16:D8 light cycle with supplementary lighting for 4 days.

We collected adult $C$. septempunctata from natural habitats close to Uppsala, Sweden $\left(59^{\circ} 47^{\prime} \mathrm{N}, 17^{\circ} 39^{\prime} \mathrm{E}\right)$, and reared them through several generations before they were used in experiments. They were reared in culture in cages with $R$. padi on barley (cv. Golf) and flowering oilseed rape, Brassica napus L. as a source of pollen at $21 \pm 1^{\circ} \mathrm{C}$, a photoperiod of $16 \mathrm{~L}: 8 \mathrm{D}$, and relative humidity $60 \pm 10 \%$. Insects for experiments were adults. Due to constraints on completing behavioural bioassays within a fixed timeframe, it was not feasible to test males and females separately and achieve a satisfactory level of replication, so we did not explicitly build this into our experimental design. However, randomly sampling groups of ladybirds from our cultures and sexing them according to Baungaard (1980) consistently gives approximately equal numbers of males and females.

Ladybird experience on aphid-infested plants

Ladybirds were confined individually to pots of 10 aphidinfested plants of a particular barley cultivar by enclosing the pot in a perforated plastic bag. The bags were kept in a glasshouse at $20 \pm 2{ }^{\circ} \mathrm{C}$, with a L16:D8 light cycle, and plants of different cultivars were kept at least $2 \mathrm{~m}$ apart. In all experiments, prior to the start of the experience treatment, ladybirds were removed from culture and kept 
without access to food for $48 \mathrm{~h}$ in a glasshouse at $20 \pm 2^{\circ} \mathrm{C}$, with a L16:D8 light cycle with supplementary lighting. Water was provided in a glass tube plugged with cotton wool. At the end of each experience treatment, ladybirds were again held under similar conditions without food for an overnight period of approximately $16 \mathrm{~h}$ prior to olfactory bioassay. We conducted a series of experiments to examine the effect of experience on ladybird olfactory preference and whether associative learning occurred.

\section{Olfactometer bioassay}

C. septempunctata was tested in a two-way airflow olfactometer with flow rate $300 \mathrm{ml} / \mathrm{min}$, as previously described (Glinwood et al. 2009; Ninkovic et al. 2001). The bioassay provides a measure of behavioural attraction and/or arrestment in response to an odour cue. An adult was placed in the olfactometer for $10 \mathrm{~min}$ and its position recorded at 2-min intervals. We used the observation frequency method (Glinwood et al. 2009; Ninkovic et al. 2001), which gives a reliable measure irrespective of whether the behaviour is characterised by frequent short visits or few long visits in the olfactometer arm. The accumulated number of observations in the arm zones after ten observations was regarded as one replicate. If an insect did not move between three consecutive observations (was motionless) we considered it to be unresponsive and discarded the replicate and started a new one with a fresh insect. Pots with plants were contained in Perspex cages $(12 \times 12 \times 40 \mathrm{~cm})$ with a hole $(8 \mathrm{~cm}$ diameter $)$ in the side to allow air to enter (Glinwood et al. 2009; Ninkovic et al. 2001). Cages were connected to opposite arms of the olfactometer using Teflon tubing and plants were not visible to ladybirds.

Each experiment was replicated with 20-21 individual ladybirds, using five olfactometers simultaneously with the positions of the treatment arms alternating. Thus, five separate pots of infested plants of the two cultivars being tested were used for each experiment. Data were analysed with Wilcoxon matched pairs tests in the Statistica software (Statsoft version published 2005). We determined the underlying ladybird olfactory preferences for aphid-infested barley cultivars through the following olfactory choice tests: (1) infested Lina versus uninfested Lina; (2) infested Barke versus uninfested Barke; and (3) infested Barke versus infested Lina. The equivalent comparisons were carried out for the cultivars Frieda and Prestige.

\section{Experiment 1. Does feeding on aphid-infested plants affect ladybird olfactory preference}

Olfactory learning in predatory arthropods has been found to require experience over several days (De Boer and Dicke
2006), although periods of $24 \mathrm{~h}$ and less have also been reported (De Boer et al. 2005). We therefore started by giving ladybirds a 5-day experience on either one of two aphid-infested barley cultivars. In separate experiments, cultivar Barke was compared with Lina, and cultivar Frieda with Prestige, since preliminary analysis had suggested that both qualitative and quantitative differences in volatile emissions existed in these pairwise comparisons.

\section{Experiment 2. Is a $24 \mathrm{~h}$ experience sufficient to affect ladybird olfactory preference?}

Having established that a 5-day experience can modify olfactory preferences, we repeated the above experiment with a 24-h experience in both cultivar pairs. Having established that a 24-h experience was sufficient to modify ladybird olfactory preferences, we performed further experiments with a 24-h experience in the Barke and Lina pairing.

\section{Experiment 3. Does experience affect the underlying preference for infested plants over uninfested plants?}

Ladybirds were experienced on either Lina or Barke as in Experiment 2. Half the ladybirds from each experience cultivar were tested against the odour of infested Lina compared with uninfested Lina and half were tested against the odour of infested Barke against uninfested Barke.

\section{Experiment 4. Can the 'learnt' olfactory preference be 'forgotten'?}

Ladybirds were experienced as in Experiment 2 above; however, after the first olfactory testing, they were returned individually to glass jars closed with permeable net. They were left here for 4 days with access to water in plastic tubes closed with cotton wool and were fed soft whey cheese (mesost; Fjällbrynt, Sweden), which we have previously found to be an excellent food source supporting ladybird growth and development. Thus, insects did not experience nutritional stress but were not exposed to aphids or plants. Ladybirds were then starved overnight (16 h) before being re-tested in the olfactometer.

\section{Experiment 5. Is temporal pairing of odour and food necessary and are odours from aphids involved?}

We de-coupled the plant and aphid stimuli, by first giving ladybirds 24-h experience on uninfested Barke or Lina, followed immediately by allowing them to feed on aphids that had previously been reared on the same cultivar in a Petri dish. Ladybirds were enclosed individually in Petri dishes containing 100 aphids that had been reared for 
4 days on the relevant cultivar. Preliminary tests showed this number of aphids to be in surplus to the ladybirds' nutritional requirements. Ladybirds thus experienced plant volatiles from the barley cultivar (without reward) and would have subsequently encountered cues such as aphid alarm pheromone, chemicals on the aphid body surface and volatiles from aphid honeydew during the feeding period. After $24 \mathrm{~h}$ of feeding, ladybirds were starved overnight $(16 \mathrm{~h})$ before olfactory testing.

\section{Experiment 6. Are olfactory responses modified by sensitisation?}

In order to test whether experience on aphid infested plants affected subsequent olfactory preference via sensitisation, we confined ladybirds on aphid-infested Barke or Lina as in Experiment 2, but they were prevented from eating aphids. Individual ladybirds were confined in plastic tubes $(5 \times 2 \mathrm{~cm}$ diameter) with two open ends closed with airpermeable net. Tubes were placed horizontally on the soil at the base of the plants, so that falling aphid honeydew could not enter. Ladybirds thus experienced volatiles associated with aphid-infested plants but with no food reward. After a 24-h experience, ladybirds were starved overnight $(16 \mathrm{~h})$ before olfactory testing.

\section{Collection and identification of plant volatiles}

Volatiles from aphid-infested barley plants were collected by air-entrainment (Agelopoulos et al. 1999) during the same period of the year that the behavioural experiments were done. The whole pot with 10 plants was placed inside a polyester (PET) cooking bag (Stewart-Jones and Poppy 2006) $(60 \times 55 \mathrm{~cm}$, Toppits; Melitta, Sweden $)$. A glass liner containing $50 \mathrm{mg}$ of the molecular adsorbent Tenax TA (Atas GL Intl., Veldhoven, Netherlands) was inserted through a small hole cut in one corner of the bag. A positive pressure push-pull system was used, with charcoal-filtered air pushed in through a Teflon tube inserted through a small hole in the bottom of the bag, at $600 \mathrm{ml} /$ $\mathrm{min}$ and pulled out over the adsorbent at $400 \mathrm{ml} / \mathrm{min}$. Bags were baked in an oven at $140^{\circ} \mathrm{C}$ for $2 \mathrm{~h}$ immediately prior to the entrainment. Charcoal filters and Tenax tubes were baked at 180 and $220^{\circ} \mathrm{C}$, respectively, under a flow of nitrogen for $16 \mathrm{~h}$. Plants of each cultivar were prepared and infested with aphids as described above for the behavioural experiments. After 4 days infestation, volatiles were collected for a period of $48 \mathrm{~h}$. Six replicates (pots) of cultivars Frieda, Prestige, Barke and Lina were carried out along with three controls consisting only of soil in pots.

Volatiles were analysed by gas chromatography-mass spectrometry (GC/MS) on an Agilent 7890N (Agilent Technologies) GC coupled to an Agilent 5975C mass selective detector (electron impact $70 \mathrm{eV}$ ). The GC was equipped with an HP-1 column (100\% dimethyl polysiloxane, $50 \mathrm{~m}, 0.32 \mathrm{~mm}$ i.d. and $0.52 \mu \mathrm{m}$ film thickness; J\&W Scientific, USA), and fitted with an Optic 3 thermal desorption system (Atas GL Intl.). The liner containing the Tenax with absorbed volatiles was placed directly into the injector and volatiles were thermally desorbed starting at $30^{\circ} \mathrm{C} / 0.5 \mathrm{~min}$, and rising at $30^{\circ} \mathrm{C} / \mathrm{s}$ to $250^{\circ} \mathrm{C}$. The $\mathrm{GC}$ temperature program was $30^{\circ} \mathrm{C} / 4 \mathrm{~min}, 5^{\circ} \mathrm{C} / \mathrm{min}$ to $150^{\circ} \mathrm{C} /$ $0.1 \mathrm{~min}, 10^{\circ} \mathrm{C} / \mathrm{min}$ to $250^{\circ} \mathrm{C} / 15 \mathrm{~min}$, using helium as carrier with a flow rate of $1.3 \mathrm{ml} / \mathrm{min}$. Volatile compounds were identified by comparison against a commercially available library (NIST 08) and by comparison of mass spectra and retention indices with commercially available authentic standards (Sigma-Aldrich, Sweden). Compounds were quantified using four-point response curves constructed using authentic standards. (E)-ocimene was quantified using a commercially available standard containing (z)-ocimene. Only compounds appearing in collections from plants with aphids and not from pots with soil were quantified. Mean amounts of volatiles from infested plants of Frieda were compared with those from Prestige, and those from Barke with Lina. Data were square roottransformed and analysed by ANOVA in Statistica (Statsoft).

\section{Results}

Olfactory response of ladybirds to aphid infested barley cultivars

Ladybirds were observed significantly more often in the olfactometer arm containing the odour of aphid-infested barley cultivar Barke compared with uninfested Barke, and aphid-infested cultivar Lina compared with uninfested Lina (Table 1). Preference for the odour of infested plants over uninfested plants was also found for the cultivars Frieda and Prestige (Table 1). Ladybirds showed no behavioural preference when presented with a choice between the odour of infested Barke and infested Lina or the odour of infested Frieda and infested Prestige (Table 1).

Effect of experience on olfactory response of ladybirds to aphid infested barley cultivars

Experiment 1. Does feeding on aphid-infested plants affect ladybird olfactory preference?

Ladybirds experienced for 5 days on aphid-infested plants of a particular cultivar preferred the odour of infested plants of the same cultivar, in both pair wise cultivar comparisons Barke versus Lina and Frieda versus Prestige 
Table 1 Olfactory response of seven-spot ladybird Coccinella septempunctata to barley cultivars Lina, Barke, Frieda and Prestige uninfested or infested with 20 aphids/plant for 4 days

\begin{tabular}{|c|c|c|c|c|}
\hline \multirow[t]{2}{*}{ Olfactory choice } & \multirow{2}{*}{$\begin{array}{l}\text { Number of visits in olfactometer } \\
\text { arm }[\text { mean }( \pm \text { SE) }]\end{array}$} & \multirow[t]{2}{*}{$n$} & \multicolumn{2}{|c|}{ Wilcoxon test } \\
\hline & & & $Z$ & $P$ \\
\hline Lina infested & $5.45(0.32)$ & 20 & 2.66 & 0.007 \\
\hline Lina uninfested & $3.45(0.30)$ & & & \\
\hline Barke infested & $5.21(0.26)$ & 20 & 2.67 & 0.007 \\
\hline Barke uninfested & $3.57(0.31)$ & & & \\
\hline Lina infested & $4.01(0.25)$ & 21 & 1.63 & 0.10 \\
\hline Barke infested & $4.90(0.26)$ & & & \\
\hline Frieda infested & $5.20(0.29)$ & 20 & 2.84 & 0.005 \\
\hline Frieda uninfested & $3.35(0.31)$ & & & \\
\hline Prestige infested & $5.55(0.38)$ & 20 & 2.93 & 0.003 \\
\hline Prestige uninfested & $3.20(0.32)$ & & & \\
\hline Frieda infested & $4.14(0.26)$ & 21 & 0.83 & 0.41 \\
\hline Prestige infested & $4.47(0.27)$ & & & \\
\hline
\end{tabular}

(Fig. 1a). Experiment 2 showed that the preference is formed after experience lasting $24 \mathrm{~h}$ or less in both cultivar combinations (Fig. 1b).

\section{Experiment 3. Does experience affect the underlying preference for infested plants over uninfested plants?}

Ladybirds experienced on aphid-infested Barke showed an olfactory preference for the odour of infested Barke over uninfested Barke and those experienced on Lina preferred infested Lina over uninfested Lina (Fig. 2). However, ladybirds did not show a preference for infested plants over uninfested plants when experienced on a different cultivar, suggesting the underlying behavioural response to the odour of infested plants was modified when the odour profile did not match that acquired during the feeding experience.

\section{Experiment 4. Can the 'learnt' olfactory preference be 'forgotten'?}

Confining ladybirds for $24 \mathrm{~h}$ on aphid-infested Barke or Lina resulted in ladybirds exhibiting a preference for the odour of infested plants of the same cultivar as in Experiment 2. When the ladybirds were re-tested after a period of 4 days without access to plants/aphids, they showed no preference for the odour of the two cultivars (Fig. 3), suggesting a return to the pre-experience behaviour.

\section{Experiment 5. Is temporal pairing of the odour and food necessary and are odours from aphids involved?}

When ladybirds were confined on uninfested plants of cultivar Barke for $24 \mathrm{~h}$, and then allowed to feed for a further $24 \mathrm{~h}$, in the absence of the plant, on aphids that had developed on Barke, they showed no preference when presented with a choice between the odour of aphidinfested Barke or Lina [mean $( \pm \mathrm{SE})$ observations in the odour of Barke 4.55 (0.33) and Lina 4.11 (0.31) Wilcoxon test $Z=0.56, P=0.57, n=20]$. Ladybirds confined on uninfested Lina for $24 \mathrm{~h}$, and then allowed to feed for a further $24 \mathrm{~h}$, in the absence of the plant, on aphids that had developed on Lina also showed no preference between the odour of aphid-infested Barke or Lina [mean $( \pm \mathrm{SE})$ observations in the odour of Barke 4.40 (0.38) and Lina 4.35 (0.36), Wilcoxon test $Z=0.05, P=0.96$, $n=20]$. This suggests that temporal pairing of odour and food is necessary for the preference to form, and that the odour of aphids or their by-products alone were not responsible.

\section{Experiment 6. Are olfactory responses modified} by sensitisation?

When ladybirds were confined on aphid-infested plants of cultivar Barke for $24 \mathrm{~h}$, but prevented from contacting aphids or aphid-products, they showed no preference when presented with a choice between the odour of aphidinfested Barke or Lina [mean $( \pm \mathrm{SE})$ observations in the odour of Barke 4.15 (0.41) and Lina 4.10 (0.38), Wilcoxon test $Z=0.15, P=0.87, n=20]$. Ladybirds confined on aphid-infested plants of cultivar Lina for $24 \mathrm{~h}$ without contacting aphids or aphid-products also showed no preference between the odour of aphid-infested Barke or Lina [mean $( \pm \mathrm{SE})$ observations in the odour of Barke 4.10 (0.34) and Lina $4.63(0.39)$, Wilcoxon test $Z=0.63$, $P=0.53, n=20]$. This suggests that associative learning rather than sensitisation is involved. 
(a) ladybirds from Frieda

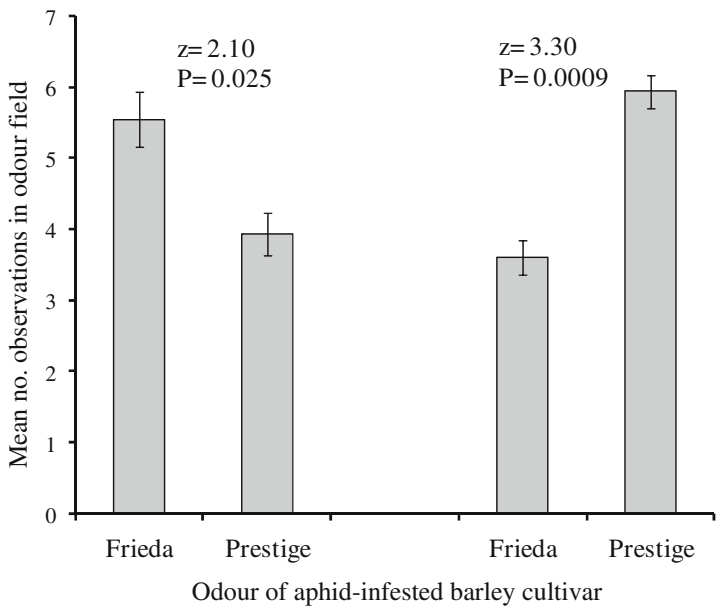

(b) ladybirds from Frieda

ladybirds from Prestige

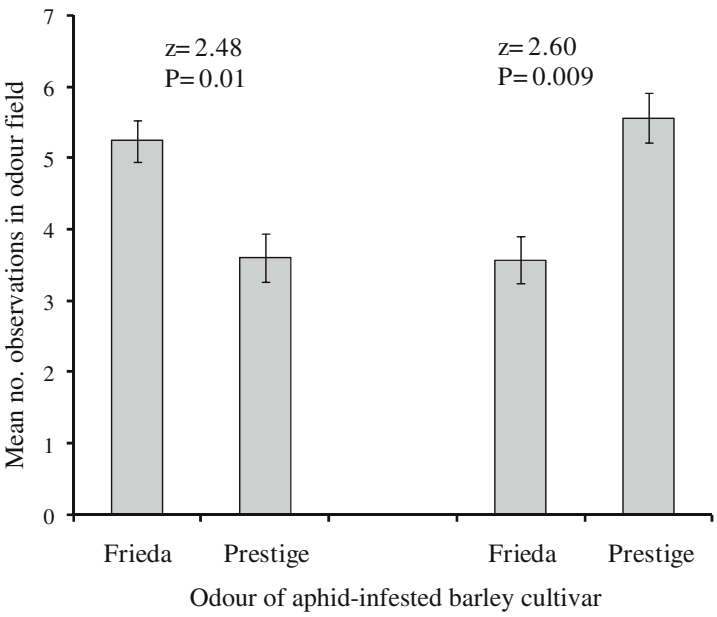

Fig. 1 Olfactory response of seven-spot ladybird Coccinella septempunctata to aphid-infested barley cultivars after feeding experience on one cultivar for a 5 days and $\mathbf{b} 1$ day (Experiments 1

Volatile profiles of aphid-infested barley cultivars

Significant differences in the volatile profiles of aphidinfested plants occurred between Frieda and Prestige and between Barke and Lina (Fig. 4a). Several sesquiterpenes were released by Prestige but not Frieda, and there were quantitative differences in several other compounds. The compound $(E)-\beta$-farnesene is the aphid alarm pheromone and is most likely of aphid rather than plant origin (Dewhirst and Pickett 2010). It was found in significantly higher amounts in Prestige. The monoterpenes $(z)-\beta$-ocimene and $(E)-\beta$-ocimene were released by Barke but not Lina, and the sesquiterpene $\alpha$-humulene was released by Lina but not Barke (Fig. 4b). There were quantitative differences in several other compounds. ladybirds from Barke ladybirds from Lina

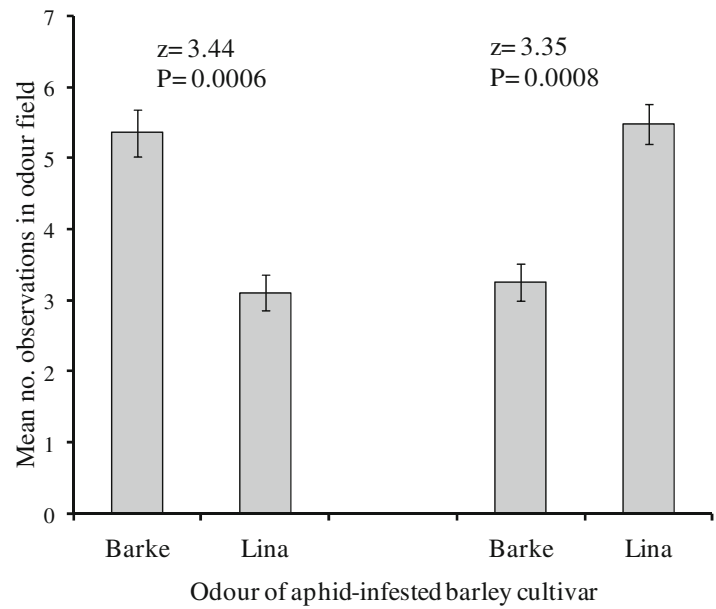

ladybirds from Barke

ladybirds from Lina

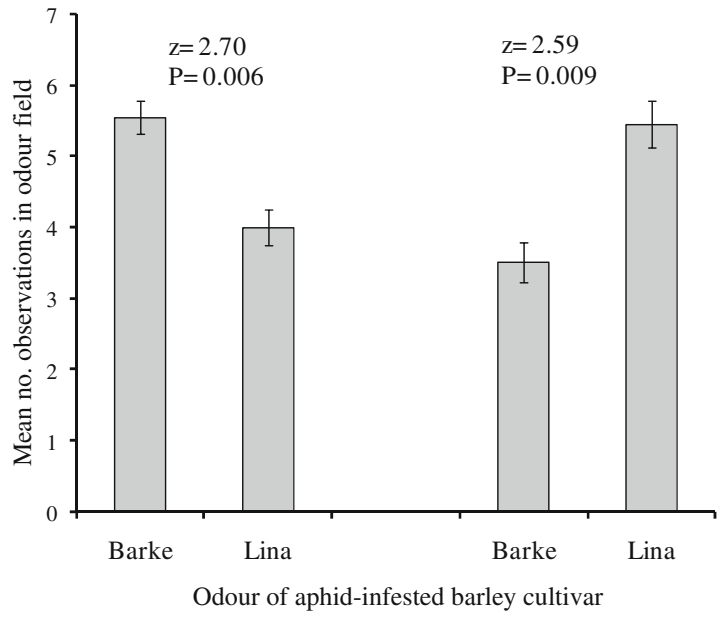

and 2). Mean number of observations in olfactometer arm $( \pm \mathrm{SE})$, values from Wilcoxon test, $n=21$ for each treatment in (a) and 22 for each treatment in $(\mathbf{b})$

\section{Discussion}

The olfactory preference of C. septempunctata was modified by experience, and the insect learned to discriminate between the odours of aphid-infested plants of different genotypes of the same host plant species, barley. There is considerable evidence for learning in arthropods (Dukas 2008; Papaj and Prokopy 1989), but whereas numerous examples of olfactory learning in parasitoids have been documented (Vet and Dicke 1992), there are fewer examples from predators (De Boer and Dicke 2006; Drukker et al. 2000). Although other types of learning have been shown in coccinellids (Boivin et al. 2010; Dejean et al. 2003; Ettifouri and Ferran 1993; Guershon and Gerling 2006; Houck 1986) our results are the first evidence for olfactory learning. 
Fig. 2 Olfactory response of seven-spot ladybird Coccinella septempunctata to aphid cultivars Barke and Lina after a 1-day feeding experience on the opposite cultivar (Experiment 3 ). Mean number of observations in olfactometer arm $( \pm$ SE $)$, values from Wilcoxon test, $n=20$ for each treatment infested and uninfested barley

ladybirds from Lina

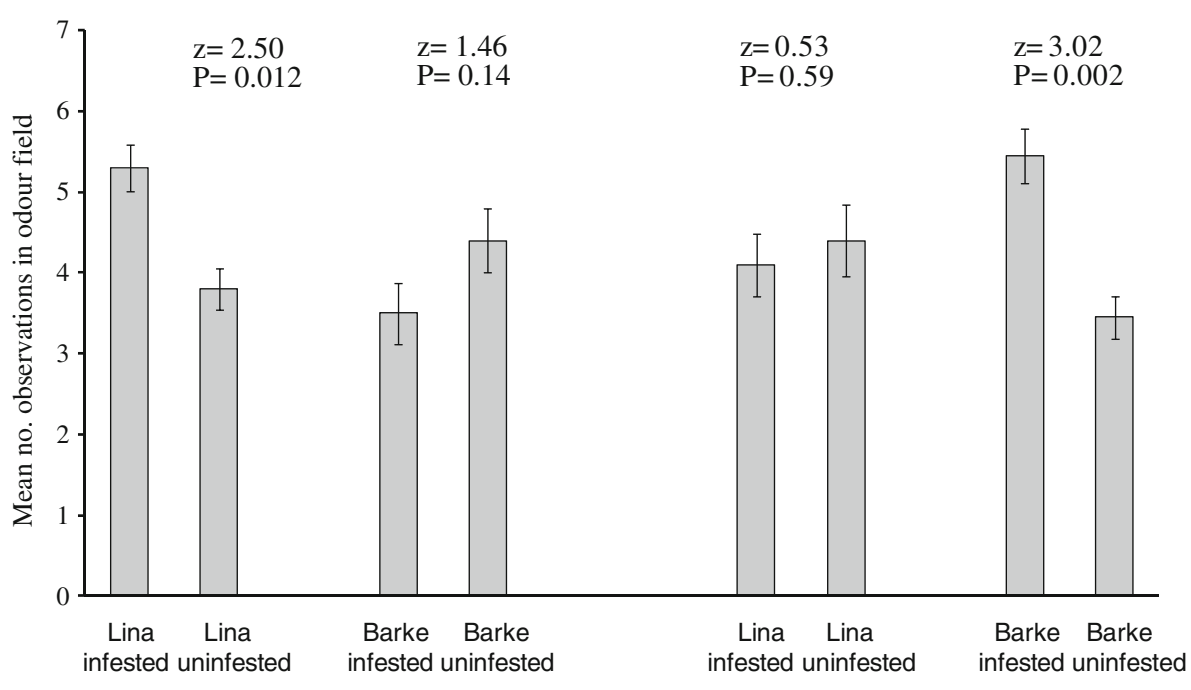

Odour of barley cultivar

Fig. 3 Olfactory response of seven-spot ladybird Coccinella septempunctata to aphidinfested barley cultivars 1 and 4 days after feeding experience on one cultivar (Experiment 4). Mean number of observations in olfactometer arm $( \pm \mathrm{SE})$, values from Wilcoxon test, $n=20$ for each treatment

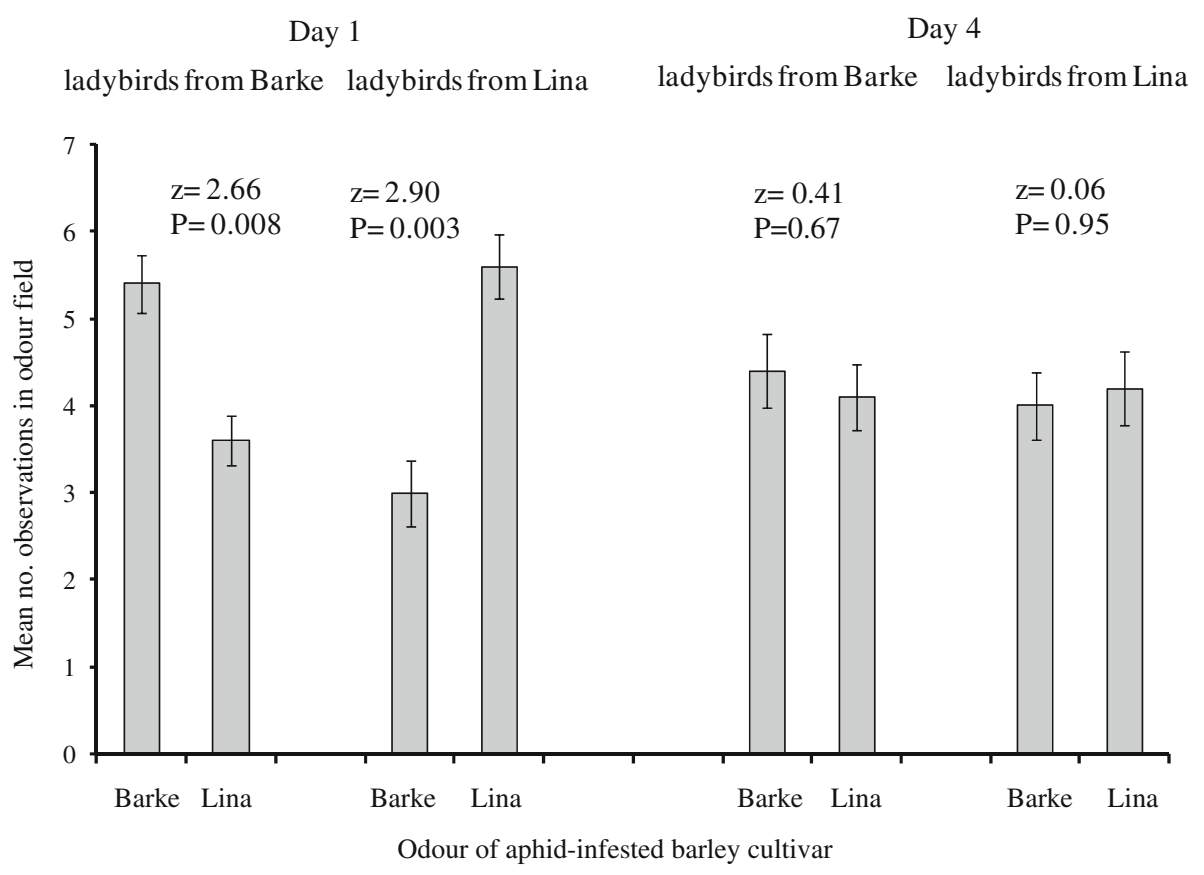

Papaj and Prokopy (1989) proposed that learning occurs when an insect's behaviour (1) changes in a repeatable way as a consequence of experience, (2) changes gradually with continued experience, and (3) wanes in the absence of continued experience of the same type. Our results fulfil criteria (1) (Experiments 1 and 2) and (3) (Experiment 4), whereas (2) was not explicitly tested due to the difficulty of demonstrating 'strength of response' in the olfactometer. Experiment 5 suggests that temporal pairing of odour and conditional stimulus (aphids) was necessary; however, this is not conclusive since we do not know whether ladybirds 'forgot' the plant odour in the intervening period, and the odour blend experienced from uninfested plants was different to that produced by aphid infested plants (data not shown). However, Experiment 5 does suggest that ladybirds learnt plant odours rather than the odour from aphids or aphid by-products. If olfactory sensitisation were involved, simple exposure to the odour blend without the reinforcing conditional stimulus of food would have affected the odour preference, and Experiment 6 suggests that this was not the case. Thus, we conclude that the most likely mechanism was associative learning.

C. septempunctata inhabits a complex botanical landscape in which it forages for high quality food sources. 
(a)

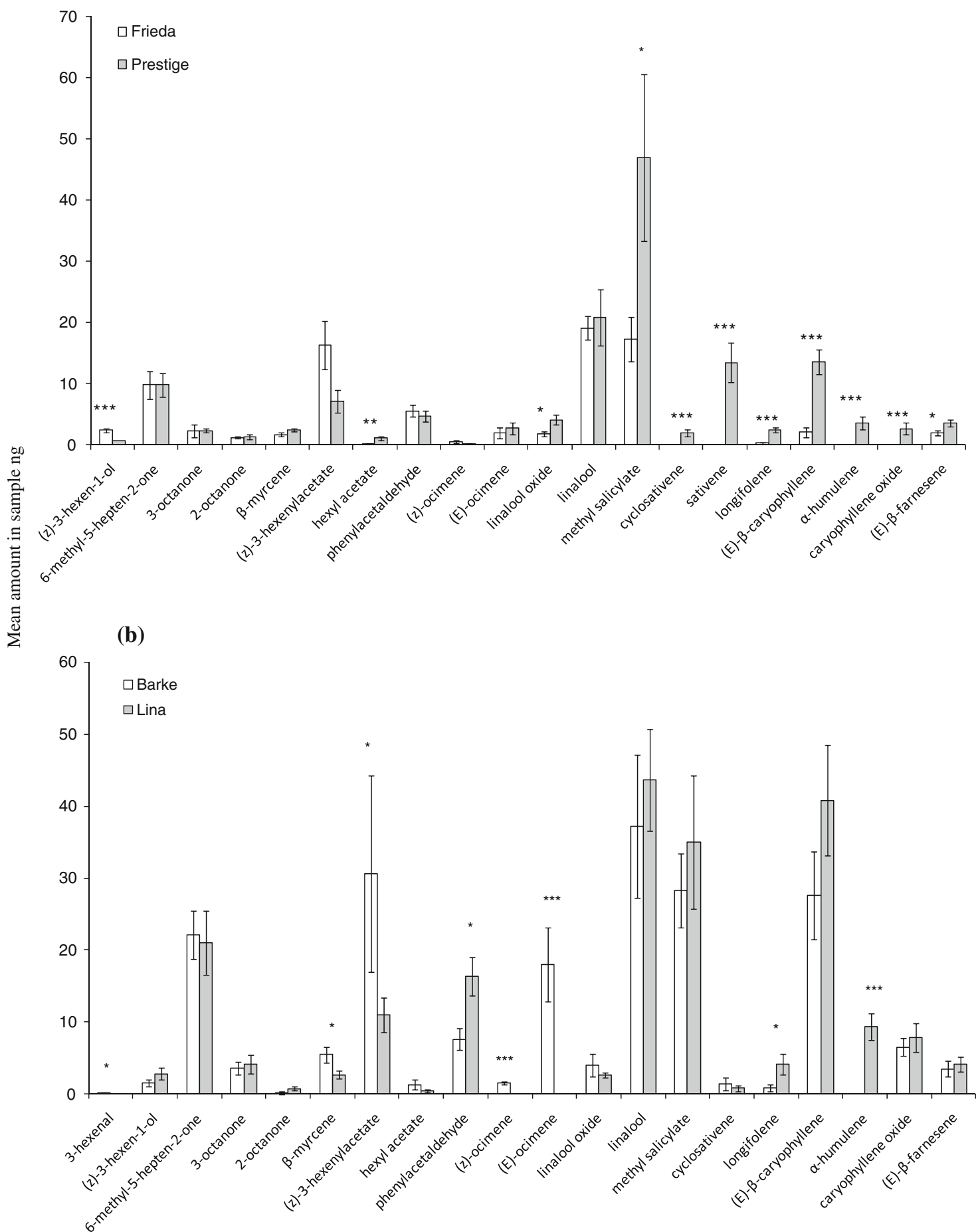

Fig. 4 Volatile profiles of aphid-infested barley cultivars Frieda compared with Prestige and Barke compared with Lina. Mean amounts ng $( \pm \mathrm{SE})$ in $48 \mathrm{~h}$ volatile collection from 10 infested plants.

Although the diet is broad, encompassing pollen and a range of small insects, aphids are considered a vital, high quality food needed to optimise fitness and fecundity (Evans et al. 1999; Hodek and Honek 1996). However, the
Significance from ANOVA on transformed means $* P<0.05$, $* * P<0.01, * * * P<0.001$

occurrence of aphids in both time and space varies in a way that is partly, but not completely, predictable by habitat and season (Ferran and Dixon 1993). Even in agricultural crops such as barley, where this ladybird is commonly found, the 
distribution of aphids may be patchy and short lived (Pettersson 1994). Once aphids are encountered, it may favour a foraging ladybird both to remain in the food patch until it is fully exploited and to continue searching for the same food source in the vicinity. Theory suggests that learning should be more evident in generalist insects with a broad diet than in specialists (Vet and Dicke 1992). Although $C$. septempunctata is a polyphagous predator with a wide range of potential foods, it can also be considered to specialise temporarily on aphid prey when and where it is available. The ability to learn, and for the learnt response to wane when no longer relevant, may allow ladybirds to maximise the effectiveness of this temporary specialisation. Olfactory learning may be part of a suite of behaviours that benefit ladybird foraging. Learning and adaptation appear to contribute to optimal handling of prey items by ladybirds (Boivin et al. 2010; Dejean et al. 2003; Ettifouri and Ferran 1993; Guershon and Gerling 2006; Houck 1986; Rana et al. 2002), and odour learning could help to maximise the time spent in patches where experience of high quality prey has already been gained.

Ladybirds responded to the odour of aphid-infested plants, as has been reported previously (Bahlai et al. 2008; Hatano et al. 2008; Ninkovic et al. 2001; Zhu and Park 2005). Although inexperienced ladybirds preferred the odour of an aphid-infested barley cultivar over uninfested plants of the same cultivar, feeding experience on a different cultivar caused this preference to disappear. This suggests that the underlying response was overridden by the novel, learnt response because the learnt stimulus was different to the underlying one. It is also possible that experience increases the strength of the behavioural response to the learnt stimulus, but this is difficult to demonstrate with the bioassay used. However, the change in preference appears to be, at least partly, mediated by a waning of response to the non-experienced stimulus. This may occur if the insect has an olfactory template against which novel cues are acquired and compared. In the absence of experience, an innate template may be used; however, learning may update and modify the current template, with a full behavioural response dependent on the strength of the match. In the absence of repeated experience with the stimulus, the template may then revert to the innate condition.

We found qualitative and quantitative differences between the volatile profiles of aphid-infested Frieda and Prestige and between aphid-infested Barke and Lina. Differences in herbivore-induced volatiles has been found between different cultivars of other crop plant species (Åhman et al. 2010; Degen et al. 2004; Loughrin et al. 1995; Takabayashi et al. 1991), although it is not yet clear if and how these are linked to plant genotype. In our study, we did not attempt to remove aphids or their products before collecting volatiles, nor did we isolate green biomass from the rhizosphere. This was because we wanted to reveal the volatile blends that ladybirds would have been exposed to when feeding on aphid-infested plants. Thus, while caution is required in linking the volatile blends to plant genotype, the data show that there was a basis for ladybirds to discriminate between the cultivars in both pairwise comparisons. We did not determine, however, which substances ladybirds can detect and respond to. It is possible that ladybirds learnt to discriminate the volatile blends based only on a few, or even single, compounds. For example, the predatory bug Anthocoris nemoralis learnt to discriminate odour blends from prey-infested and uninfested trees based on the presence of a single component, methyl salicylate (Drukker et al. 2000), and honey bees can learn mixtures based on a few key odorants (Reinhard et al. 2010). This is feasible in our study since, in both pairwise cultivar comparisons, at least two compounds were unique to one cultivar. Alternatively, the odour mixture and the ratios of its components may be perceived as having a unique character, different to that of its individual components alone (Chandra and Smith 1998).

To determine the chemical basis of the discrimination it is necessary to study the electrophysiological responses of ladybird antennae and screen individual substances and blends for behavioural activity. This was outside the scope of our investigation but will be addressed in future studies. However, arthropods can discriminate complex mixtures of volatiles, and the relevance of variation in the odour blends released by plants for the orientation of phytophagous and predatory insects is being increasingly recognised (Pareja et al. 2009; Webster et al. 2010). The importance of odour blends has also been demonstrated for insect learning (Fukushima et al. 2002; van Wijk et al. 2008).

It has been pointed out that caution is needed in ascribing adaptive value to learning behaviour in insects, particularly when demonstrated in the laboratory (Papaj and Prokopy 1989). There may be scenarios in which learning is disadvantageous, such as when it prevents an insect exploiting a suitable but less preferred resource. The costs and benefits of the type of learning demonstrated here could change dynamically depending on the availability of food; however, the benefits are expected to outweigh the costs over the longer term. C. septempunctata probably spent the major part of its evolution in natural rather than agricultural habitats, so the ability to learn should also be interpreted in this context. However, it is interesting to speculate that this may have provided a pre-adaptation to survival in the agricultural landscapes where this species is commonly found today. It is unclear whether a learningmediated shift in preference for the odour of different barley cultivars would itself be advantageous for 
C. septempunctata. The crop is usually grown over large areas as single cultivars, but certain cultivars may be more favourable for aphid growth and the cultivar planted may vary from farm to farm. Odour learning could help to retain the insect in a barley field while aphids are present rather than dispersing into the more botanically diverse surrounding landscape.

The value of learning food odours may vary between different parts of the life cycle, such as migration to and from hibernating sites, mating and aggregation. Since females must choose favourable oviposition sites where emerging larvae can feed on aphids, the hypothesis that odour learning ability differs between the sexes should also be tested. Nevertheless, the ability to discriminate the odour profiles of different cultivars of the same plant suggests a degree of sophistication in C. septempunctata's olfactory learning behaviour. Considering the challenges $C$. septempunctata must overcome in its search for high quality habitats and food, the ability to learn can be seen as an important adaptation. Although further studies are needed to examine the mechanisms and determine whether they have adaptive value, our study provides further evidence for the sophisticated use of odour cues by insect predators.

Acknowledgments This work was financially supported by Mistra through the PlantComMistra program. Funding for equipment from Stiftelsen Tornspiran is acknowledged.

Open Access This article is distributed under the terms of the Creative Commons Attribution Noncommercial License which permits any noncommercial use, distribution, and reproduction in any medium, provided the original author(s) and source are credited.

\section{References}

Agelopoulos NG, Hooper AM, Maniar SP, Pickett JA, Wadhams LJ (1999) A novel approach for isolation of volatile chemicals released by individual leaves of a plant in situ. J Chem Ecol 25:1411-1425

Åhman I, Glinwood R, Ninkovic V (2010) The potential for modifying plant volatile composition to enhance resistance to arthropod pests. $\mathrm{CAB}$ reviews: Perspectives in agriculture, veterinary science, Nutrition and Natural Resources 5

Bahlai CA, Welsman JA, Macleod EC, Schaafsma AV, Hallett RH, Sears MK (2008) Role of visual and olfactory cues from agricultural hedgerows in the orientation behavior of multicolored Asian lady beetle (Coleoptera: Coccinellidae). Environ Entomol 37:973-979

Baungaard J (1980) A simple method for sexing Coccinella septempunctata L. (Coleoptera: Coccinellidae). Enomol Medd 48:26-28

Boivin G, Roger C, Coderre D, Wajnberg E (2010) Learning affects prey selection in larvae of a generalist coccinellid predator. Entomol Exp Appl 135:48-55

Chandra S, Smith BH (1998) An analysis of synthetic processing of odor mixtures in the honeybee (Apis mellifera). J Exp Biol 201:3113-3121
De Boer JG, Dicke M (2006) Olfactory learning by predatory arthropods. Anim Biol 56:143-155

De Boer JG, Snoeren TAL, Dicke M (2005) Predatory mites learn to discriminate between plant volatiles induced by prey and nonprey herbivores. Anim Behav 69:869-879

Degen T, Dillmann C, Marion-Poll F, Turlings TCJ (2004) High genetic variability of herbivore-induced volatile emission within a broad range of maize inbred lines. Plant Physiol 135:1928-1938

Dejean A, Gibernau M, Lauga J, Orivel J (2003) Coccinellid learning during capture of alternative prey. J Insect Behav 16:859-864

Dewhirst SY, Pickett JA (2010) Production of semiochemical and allelobiotic agents as a consequence of aphid feeding. Chemoecology 20:89-96

Dicke M (2009) Behavioural and community ecology of plants that cry for help. Plant Cell Environ 32:654-665

Drukker B, Bruin J, Sabelis MW (2000) Anthocorid predators learn to associate herbivore-induced plant volatiles with presence or absence of prey. Physiol Entomol 25:260-265

Dukas R (2008) Evolutionary biology of insect learning. Annu Rev Entomol 53:145-160

Dukas R, Bernays EA (2000) Learning improves growth rate in grasshoppers. Proc Natl Acad Sci USA 97:2637-2640

Egas M, Sabelis MW (2001) Adaptive learning of host preference in a herbivorous arthropod. Ecol Lett 4:190-195

Ettifouri M, Ferran A (1993) Influence of larval rearing diet on the intensive searching behavior of Harmonia axyridis [Col, Coccinellidae] larvae. Entomophaga 38:51-59

Evans EW, Stevenson AT, Richards DR (1999) Essential versus alternative foods of insect predators: benefits of a mixed diet. Oecologia 121:107-112

Farina WM, Gruter C, Diaz PC (2005) Social learning of floral odours inside the honeybee hive. Proc R Soc Lond B 272:1923-1928

Ferran A, Dixon AFG (1993) Foraging behavior of ladybird larvae (Coleoptera, Coccinellidae). Eur J Entomol 90:383-402

Fukushima J, Kainoh Y, Honda H, Takabayashi J (2002) Learning of herbivore-induced and nonspecific plant volatiles by a parasitoid, Cotesia kariyai. J Chem Ecol 28:579-586

Glinwood R, Ahmed E, Qvarfordt E, Ninkovic V, Pettersson J (2009) Airborne interactions between undamaged plants of different cultivars affect insect herbivores and natural enemies. Arthropod Plant Interact 3:215-224

Guershon M, Gerling D (2006) Effects of plant and prey characteristics on the predatory behavior of Delphastus catalinae. Entomol Exp Appl 121:15-21

Guillette LM, Hollis KL, Markarian A (2009) Learning in a sedentary insect predator: antlions (Neuroptera: Myrmeleontidae) anticipate a long wait. Behav Process 80:224-232

Hatano E, Kunert G, Michaud JP, Weisser WW (2008) Chemical cues mediating aphid location by natural enemies. Eur J Entomol 105:797-806

Heil M (2008) Indirect defence via tritrophic interactions. New Phytol 178:41-61

Hodek I, Honek A (1996) Ecology of Coccinellidae. Kluwer, Dordrecht

Houck MA (1986) Prey preference in Stethorus punctum (Coleoptera, Coccinellidae). Environ Entomol 15:967-970

Leitner M, Boland W, Mithöfer A (2005) Direct and indirect defences induced by piercing-sucking and chewing herbivores in Medicago truncatula. New Phytol 167:597-606

Loughrin JH, Manukian A, Heath RR, Tumlinson JH (1995) Volatiles emitted by different cotton varieties damaged by feeding beet armyworm larvae. J Chem Ecol 21:1217-1227

Ninkovic V, Pettersson J (2003) Searching behaviour of the sevenspotted ladybird, Coccinella septempunctata-effects of plant-plant odour interaction. Oikos 100:65-70 
Ninkovic V, Al-Abassi S, Pettersson J (2001) The influence of aphidinduced plant volatiles on ladybird beetle searching behavior. Biol Control 21:191-195

Ninkovic V, Al Abassi S, Ahmed E, Glinwood R, Pettersson J (2010) Effect of within-species plant genotype mixing on habitat preference of a polyphagous insect predator. Oecologia. doi: 10.1007/s00442-010-1839-2

Papaj DR, Prokopy RJ (1989) Ecological and evolutionary aspects of learning in phytophagous insects. Annu Rev Entomol 34:315-350

Pareja M, Mohib A, Birkett MA, Dufour S, Glinwood RT (2009) Multivariate statistics coupled to generalized linear models reveal complex use of chemical cues by a parasitoid. Anim Behav 77:901-909

Pettersson J (1994) The bird cherry-oat aphid, Rhopalosiphum padi (HOM: APH.) and odours. In: Leather SR, Wyatt NA, Kidd NAC, Walters KFA (eds) Individuals, populations and patterns in ecology. Intercept, Andover, pp 3-12

Pettersson J, Ninkovic V, Glinwood R, Birkett MA, Pickett JA (2005) Foraging in a complex environment-semiochemicals support searching behaviour of the seven spot ladybird. Eur J Entomol 102:365-370

Pettersson $\mathrm{J}$ et al (2008) Chemical stimuli supporting foraging behaviour of Coccinella septempunctata L. (Coleoptera: Coccinellidae): volatiles and allelobiosis. Appl Entomol Zool 43:315-321

Rahmani H, Hoffmann D, Walzer A, Schausberger P (2009) Adaptive learning in the foraging behavior of the predatory mite Phytoseiulus persimilis. Behav Ecol 20:946-950

Rana JS, Dixon AFG, Jarosik V (2002) Costs and benefits of prey specialization in a generalist insect predator. J Anim Ecol $71: 15-22$

Reinhard J, Sinclair M, Srinivasan MV, Claudianos C (2010) Honeybees learn odour mixtures via a selection of key odorants. Plos One 5:14

Sarmento RA, Venzon M, Pallini A, Oliveira EE, Janssen A (2007) Use of odours by Cycloneda sanguinea to assess patch quality. Entomol Exp Appl 124:313-318
Shikano I, Isman MB (2009) A sensitive period for larval gustatory learning influences subsequent oviposition choice by the cabbage looper moth. Anim Behav 77:247-251

Stephens DW, Krebs JR (1986) Foraging theory. Princeton University Press, Princeton

Stewart-Jones A, Poppy GM (2006) Comparison of glass vessels and plastic bags for enclosing living plant parts for headspace analysis. J Chem Ecol 32:845-864

Stireman JO (2002) Learning in the generalist tachinid parasitoid Exorista mella Walker (Diptera: Tachinidae). J Insect Behav 15:689-706

Takabayashi J, Dicke M, Posthumus MA (1991) Variation in composition of predator-attracting allelochemicals emitted by herbivore-infested plants: relative influence of plant and herbivore. Chemoecology 2:1-6

Van Den Boom CEM, Van Beek TA, Posthumus MA, De Groot A, Dicke M (2004) Qualitative and quantitative variation among volatile profiles induced by Tetranychus urticae feeding on plants from various families. J Chem Ecol 30:69-89

van Nouhuys S, Kaartinen R (2008) A parasitoid wasp uses landmarks while monitoring potential resources. Proc R Soc Lond B 275:377-385

van Wijk M, De Bruijn PJA, Sabelis MW (2008) Predatory mite attraction to herbivore-induced plant odors is not a consequence of attraction to individual herbivore-induced plant volatiles. J Chem Ecol 34:791-803

Vet LEM, Dicke M (1992) Ecology of infochemical use by natural enemies in a tritophic context. Annu Rev Entomol 37:141-172

Webster B, Bruce T, Pickett J, Hardie J (2010) Volatiles functioning as host cues in a blend become nonhost cues when presented alone to the black bean aphid. Anim Behav 79:451-457

Zhu JW, Park KC (2005) Methyl salicylate, a soybean aphid-induced plant volatile attractive to the predator Coccinella septempunctata. J Chem Ecol 31:1733-1746 